\title{
External field control of donor electron exchange at the $\mathrm{Si} / \mathrm{SiO}_{2}$ interface
}

\author{
M.J. Calderón, ${ }^{1}$ Belita Koiller, ${ }^{2}$ and S. Das Sarma ${ }^{1}$ \\ ${ }^{1}$ Condensed Matter Theory Center, Department of Physics, \\ University of Maryland, College Park, MD 20742-4111 \\ ${ }^{2}$ Instituto de Física, Universidade Federal do Rio de Janeiro, \\ Caixa Postal 68528, 21941-972 Rio de Janeiro, Brazil
}

(Dated: July 7, 2021)

\begin{abstract}
We analyze several important issues for the single- and two-qubit operations in Si quantum computer architectures involving $\mathrm{P}$ donors close to a $\mathrm{SiO}_{2}$ interface. For a single donor, we investigate the donor-bound electron manipulation (i.e. 1-qubit operation) between the donor and the interface by electric and magnetic fields. We establish conditions to keep a donor-bound state at the interface in the absence of local surface gates, and estimate the maximum planar density of donors allowed to avoid the formation of a 2-dimensional electron gas at the interface. We also calculate the times involved in single electron shuttling between the donor and the interface. For a donor pair, we find that under certain conditions the exchange coupling (i.e. 2-qubit operation) between the respective electron pair at the interface may be of the same order of magnitude as the coupling in GaAs-based two-electron double quantum dots where coherent spin manipulation and control has been recently demonstrated (for example for donors $\sim 10 \mathrm{~nm}$ below the interface and $\sim 40 \mathrm{~nm}$ apart, $J \sim 10^{-4} \mathrm{meV}$ ), opening the perspective for similar experiments to be performed in Si.
\end{abstract}

PACS numbers: 03.67.Lx, 85.30.-z, 73.20.Hb, 85.35.Gv, 71.55.Cn

\section{INTRODUCTION}

Doped Si is a promising candidate for quantum computing ${ }^{1}$ due to its scalability properties, long spin coherence times, $, 2,3,4,5,6,7$ and the astonishing progress on $\mathrm{Si}$ technology and miniaturization in the last few decades (Moore's law). The experimental production of a working qubit depends on precise positioning (of the order of $\AA) \frac{8,9}{9}$ of donors in Si and the quantum control of the donor electrons by local gates placed over an oxide layer above the donors. The required accuracy in donor positioning has not been yet achieved, although there are increasing efforts in this direction, using top-down techniques, i.e. single ion implantation (with tens of $\mathrm{nm}$ accuracy), $, 10,11,12$ and bottom up techniques, i.e. positioning of $\mathrm{P}$ donors on a mono-hydride surface via STM (with $1 \mathrm{~nm}$ accuracy) with subsequent $\mathrm{Si}$ overgrowth $\underline{13.14}$

In the original doped $\mathrm{Si}$ based quantum computer proposal, $\stackrel{1}{,}$ illustrated in Fig. 1, the qubits are the donor nuclear spins, and the hyperfine interaction between these and the donor electron spins is used to perform single qubit operations (rotations). The strength of the hyperfine interaction is manipulated by local surface gates, the so called A-gates, which move the electron between the donor and an interface with $\mathrm{SiO}_{2}$. Exchange between neighboring donors, tuned by surface 'exchange' gates (J-gates), would control two-qubit operations. Exchange gates were originally proposed for a double quantum dot geometry in GaAs $\frac{15}{15}$ Related proposals in Si use the electron spin as qubit $16,17,18$ or the electron charge $\frac{19}{=}$ Charge coherence in $\mathrm{Si}$ is much shorter $(\sim 200 \mathrm{~ns})^{20}$ than the spin coherence $T_{2} \sim 1 \mathrm{~ms}$, which can be further enhanced by isotopic purification, $, 2,5, \underline{6}$ making spin qubits in general more attractive than charge qubits for actual implementations. On the other hand, direct detection of a sin-

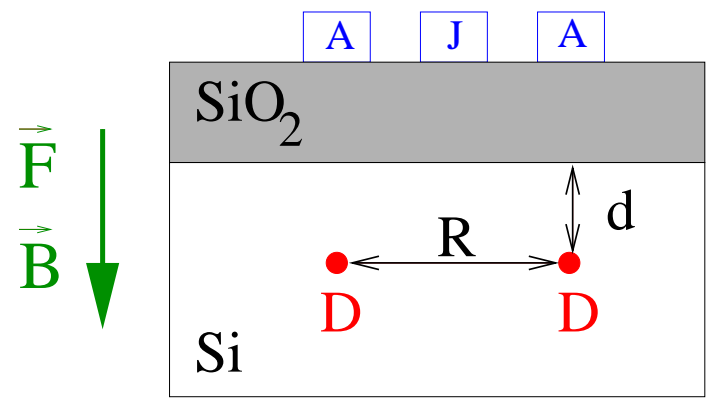

FIG. 1: (Color online) Schematic view of Kane's quantum computer. Donors D are a distance $d$ from the $\mathrm{Si} / \mathrm{SiO}_{2}(001)$ interface and a distance $R$ from each other. Surface A- and J-gates control one and two qubit operations. In the present study, we consider uniform electric $F$ and magnetic $B$ fields applied in the $z$-direction.

gle spin is a very difficult task $\underset{21,22,23}{2}$ while a fraction of a single electron charge can be easily detected with state-of-the-art single electron tunneling (SET) devices. As a result, ingenious spin-to-charge conversion mechanisms, that would allow the electron spin state to be inferred according to the absence or presence of charge detected by an SET at the surface, have been proposed, e.g. Refs. 24, 25, 26, 27.

Doped Si has two main advantages over GaAs quantum dots: (i) The much longer spin coherence times, that can be enhanced by isotopic purification (note that all isotopes of Ga and As have nuclear spins so the spin coherence time in GaAs cannot be improved via isotopic purification), and (ii) the identical Coulomb potentials created by donors as opposed to variable quantum dot well shapes produced by surface gates on a 2-dimensional 
electron gas (2DEG). Despite this latent superiority of $\mathrm{Si}$, progress in GaAs has been much faster, 23,28,29,30,31 in particular due to the fact that the electrons, being at the device surface, are easier to manipulate and detect. Another Si handicap is that the exchange between donors in bulk Si oscillates, changing by orders of magnitude when the relative position of neighboring donors changes by small distances $\sim \AA .8 .32$ This is caused by interference effects between the six degenerate minima in the Si conduction band. However, as discussed below, this degeneracy is partially lifted at the interface, thus the oscillatory behavior may not represent such a severe limitation for interface states as compared to the donor bulk states.

In the following, we analyze the manipulation of donor electrons close to a $\mathrm{Si} / \mathrm{SiO}_{2}$ interface by means of external uniform electric and magnetic fields $\underline{33}, \underline{34}$ In Sec. III we introduce the model for an isolated donor and discuss the interface and the donor ground states, which are calculated variationally. We also analyze the shuttling between the interface and the donor, including the effect of a magnetic field. In Sec. III we study the conditions to avoid the formation of a $2 \mathrm{DEG}$ at the interface, and discuss the advantages and actual feasibility of performing two-qubit operations at the interface. A summary and conclusions are given in Sec. IV]

\section{SINGLE DONOR}

\section{A. Model}

We consider initially a single donor a distance $d$ from a $\mathrm{Si} / \mathrm{SiO}_{2}$ (001) interface. As a simple model for the A-gate effects, a uniform electric field is applied in the $z$-direction, as illustrated in Fig. 1 The inter-donor distance $R$ is assumed to be large enough so that each donor can be treated as an isolated system. The conduction band of Si has six equivalent minima located along the $\Delta$ lines. As discussed below, it is reasonable to treat this system within the single-valley effective mass approximation $35,36,37,38,39$ leading to the following Hamiltonian for the donor electron

$H=T+e F z-\frac{e^{2}}{\epsilon_{\mathrm{Si}} r}+\frac{e^{2} Q}{\epsilon_{\mathrm{Si}} \sqrt{\rho^{2}+(z+2 d)^{2}}}-\frac{e^{2} Q}{4 \epsilon_{\mathrm{Si}}(z+d)}$.

We also consider an applied magnetic field along $z$ : In this case the vector potential $\mathbf{A}=B(y,-x, 0) / 2$ is included in the kinetic energy term, $T=$ $\sum_{\eta=x, y, z} \hbar^{2} /\left(2 m_{\eta}\right)\left[i \partial / \partial \eta+e A_{\eta} /(\hbar c)\right]^{2}$. The effective masses in Si are $m_{x}=m_{y}=m_{\perp}=0.191 \mathrm{~m}$, and $m_{z}=$ $m_{\|}=0.916 \mathrm{~m}$. The second term is the electric field linear potential, the third is the donor Coulomb potential, and the last two terms (with $\rho^{2}=x^{2}+y^{2}$ ) take account of the charge images of the donor and the electron, respectively. $Q=\left(\epsilon_{\mathrm{SiO}_{2}}-\epsilon_{\mathrm{Si}}\right) /\left(\epsilon_{\mathrm{SiO}_{2}}+\epsilon_{\mathrm{Si}}\right)$, where $\epsilon_{\mathrm{Si}}=11.4$ and $\epsilon_{\mathrm{SiO}_{2}}=3.8$. In this case $Q<0$ and, therefore, the

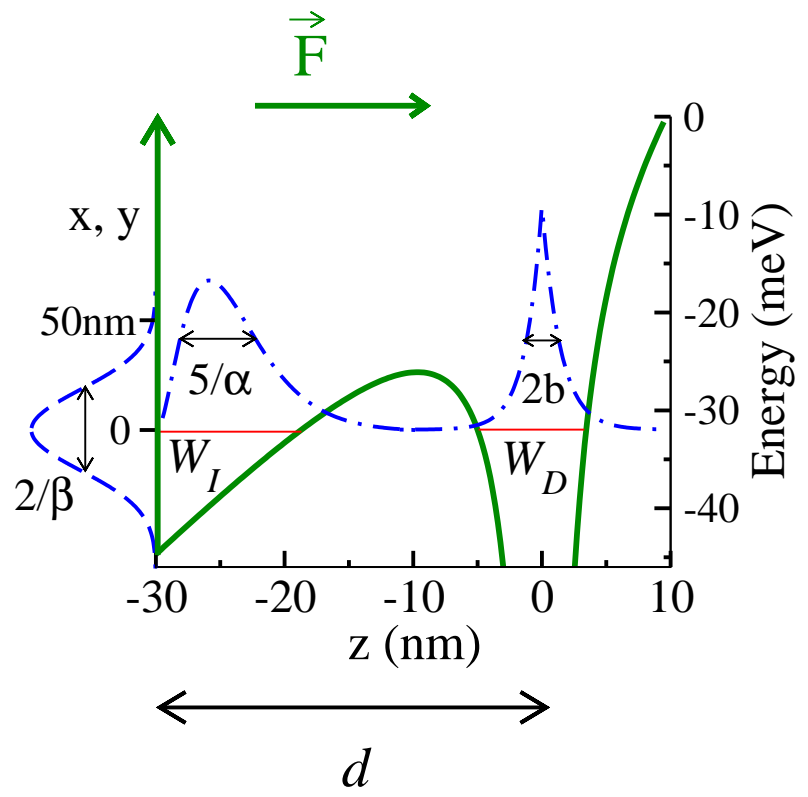

FIG. 2: (Color online) Double-well potential formed by the donor nuclear charge $\left(\mathrm{W}_{D}\right)$, the applied electric field, and the interface with the oxide (considered to be $\infty$, i.e., impenetrable). The interface well $\mathrm{W}_{I}$ consists of the electric field and the interface in the $z$-direction and also includes the nuclear charge and its image in the $x y$-plane [(see Fig. 3(a)]. The dashed lines represent the decoupled ground eigenstates in each well $\psi_{I}$ and $\psi_{D}$. The thin horizontal lines indicate the expectation value of the energy in each well. The potential, wave-functions and energies depicted correspond to $d=30 \mathrm{~nm}$ and $F=13.5 \mathrm{kV} / \mathrm{cm} \approx F_{\mathrm{c}}(30 \mathrm{~nm})$.

images have the same sign as the originating charges. In rescaled atomic units, $a^{*}=\hbar^{2} \epsilon_{S i} / m_{\perp} e^{2}=3.157 \mathrm{~nm}$ and $R y^{*}=m_{\perp} e^{4} / 2 \hbar^{2} \epsilon_{S i}^{2}=19.98 \mathrm{meV}$, and the Hamiltonian is written

$$
\begin{aligned}
H & =-\frac{\partial^{2}}{\partial x^{2}}-\frac{\partial^{2}}{\partial y^{2}}-\gamma \frac{\partial^{2}}{\partial z^{2}}+\frac{1}{4} \mu^{2} \rho^{2}+i \mu\left(y \partial_{x}-x \partial_{y}\right) \\
& +\kappa e F z-\frac{2}{r}+\frac{2 Q}{\sqrt{\rho^{2}+(z+2 d)^{2}}}-\frac{Q}{2(z+d)}
\end{aligned}
$$

where $\gamma=m_{\perp} / m_{\|}, \mu^{2}=a^{* 4} / \lambda_{B}^{4}$ with $\lambda_{B}=\sqrt{\hbar / e B}$ the magnetic length, $\kappa=3.89 \times 10^{-7} \epsilon_{S i}^{3}\left(\mathrm{~m} / \mathrm{m}_{\perp}\right)^{2} \mathrm{~cm} / \mathrm{kV}$, and the electric field $F$ is given in $\mathrm{kV} / \mathrm{cm}$.

The system under study consists of a shallow donor, $\mathrm{P}$ in particular, immersed in $\mathrm{Si}$ a distance $d$ from a $\mathrm{SiO}_{2}$ barrier, which we assume to be infinite (impenetrable). When no external field is present, the electron is bound to the donor potential well $W_{D}$. When an electric field $F$ is applied in the $z$-direction, a triangular well $W_{I}$ is formed next to the interface. The interface well $W_{I}$ also includes the donor and its image Coulomb potentials at the interface $(z=-d)$ which, under special circumstances discussed below, are strong enough to localize the electron in the $x y$-plane. The interface well $W_{I}$ and the donor 
Coulomb potential $W_{D}$ form an asymmetric double well, as illustrated in Fig. 2 .

The Hamiltonian in Eq. (21) is solved in the basis formed by $\psi_{D}$ and $\psi_{I}$, which are the ground eigenstates of each of the decoupled wells $W_{D}$ and $W_{I}$. The Hamiltonian is written

$$
H=T+V_{I}+V_{D}+\frac{2}{\sqrt{\rho^{2}+d^{2}}}
$$

where the last term is added to avoid double counting of the impurity Coulomb potential at the interface included both in $V_{I}$ and $V_{D}$, which are defined as

$$
V_{I}=2 \frac{Q-1}{\sqrt{\rho^{2}+d^{2}}}+\kappa e F z-\frac{Q}{2(z+d)}
$$

and

$$
V_{D}=-\frac{2}{r}
$$

The first term in Eq. (4) is the sum of the donor Coulomb potential and its image charge potential at the interface.

The Hamiltonian in the non-orthogonal basis $\left\{\psi_{D}, \psi_{I}\right\}$ reads

$$
\left(\begin{array}{cc}
H_{D D} & H_{I D} \\
H_{I D} & H_{I I}
\end{array}\right)
$$

where $H_{A B}=\left\langle\psi_{A}|H| \psi_{B}\right\rangle$ with $A, B=I, D$. Diagonalization gives the two eigenstates $\Psi^{+}$and $\Psi^{-}$with eigenenergies $E^{+}$and $E^{-}$which show anticrossing behavior with a minimum gap when $H_{D D}=H_{I I}$. This point defines the characteristic field $F_{\mathrm{c}}$, illustrated in Fig. 2, which is relevant for the tunneling process discussed in detail in Sec. IID

\section{B. Interface state $\psi_{I}$}

It is convenient to write the interface potential $V_{I}$ as a sum of purely $z$ - and purely $\rho$-dependent terms:

$$
\begin{aligned}
V_{I} & =V_{I}^{z}+V_{I}^{\rho}, \\
V_{I}^{z} & =\kappa e F z-\frac{Q}{2(z+d)}, \\
V_{I}^{\rho} & =2 \frac{Q-1}{\sqrt{\rho^{2}+d^{2}}} .
\end{aligned}
$$

The $V_{I}^{z}$ component is the triangular well plus the electron image charge potential, while the $V_{I}^{\rho}$ component is the sum of the impurity and its image potential at the interface. $V_{I}^{\rho}$ is plotted in Fig. 3(a) for three different values of $d$. Curves corresponding to the parabolic approximation of the potential,

$$
V_{\text {parab }}(\rho)=(Q-1)\left(\frac{2}{d}-\frac{\rho^{2}}{d^{3}}\right),
$$

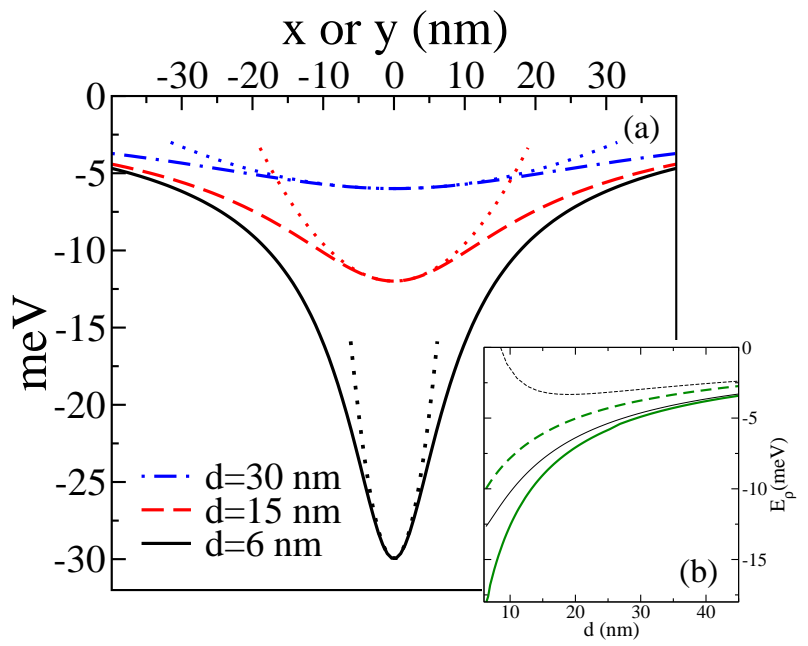

FIG. 3: (Color online) (a) Confining potential at the interface $x y$-plane due to the Coulomb attraction of the donor nucleus and its image $V_{I}^{\rho}=2(Q-1) / \sqrt{\rho^{2}+d^{2}}$. Dotted lines correspond to the harmonic approximation of the potential $V_{\text {parab }}(\rho)=(Q-1)\left(2 / d-\rho^{2} / d^{3}\right)$. (b) Ground state energy $E_{\rho}$ (solid lines) and first excited state energy $E_{\rho}^{\prime}$ at the interface (dashed lines). The thick lines correspond to the potential $V_{I}^{\rho}$ while the thin lines correspond to the parabolic approximation. As $d$ increases, the solution for the parabolic approximation approaches the one for the $V_{I}^{\rho}$ potential, as expected.

are also shown, and it is clear that the harmonic approximation works better for the larger distances $d$. Electron confinement at the interface $x y$-plane is provided by $V_{I}^{\rho}$, while the uniform electric field and the oxide confine the electron along the $z$-direction. For certain values of $d$ and $R, V_{I}^{\rho}$ is deep enough to localize the individual donor electrons (with no need of local A-gates) and keep them from forming a 2DEG at the interface: The necessary conditions are discussed in Sec. IIIA. The spacial localization of the electrons at the interface is a necessary condition for Si-based quantum computing if qubit read-out takes place at the interface $\underline{\underline{1}}$

The ground state at the interface is calculated by solving $H_{I}=T+V_{I}$ variationally with a separable trial function

$$
\psi_{I}=f(z) g(\rho) .
$$

For the $z$-part we use

$$
f(z)=\frac{\alpha^{(2 \ell+1) / 2}}{\sqrt{(2 \ell) !}}(z+d)^{\ell} e^{-\alpha(z+d) / 2}
$$

for $z>-d$. The infinite barrier at the interface is taken into account by forcing the ground state to be zero at the interface, so $f(z)=0$ for $z \leq-d$. $\alpha$ is a variational parameter that minimizes the contribution to the energy $\left\langle f(z)\left|T^{z}+V_{I}^{z}\right| f(z)\right\rangle$. The most suitable value for $\ell$ is chosen by comparing $E_{z}=\left\langle f(z)\left|T^{z}+\kappa e F z\right| f(z)\right\rangle$ and $f(z)$ 

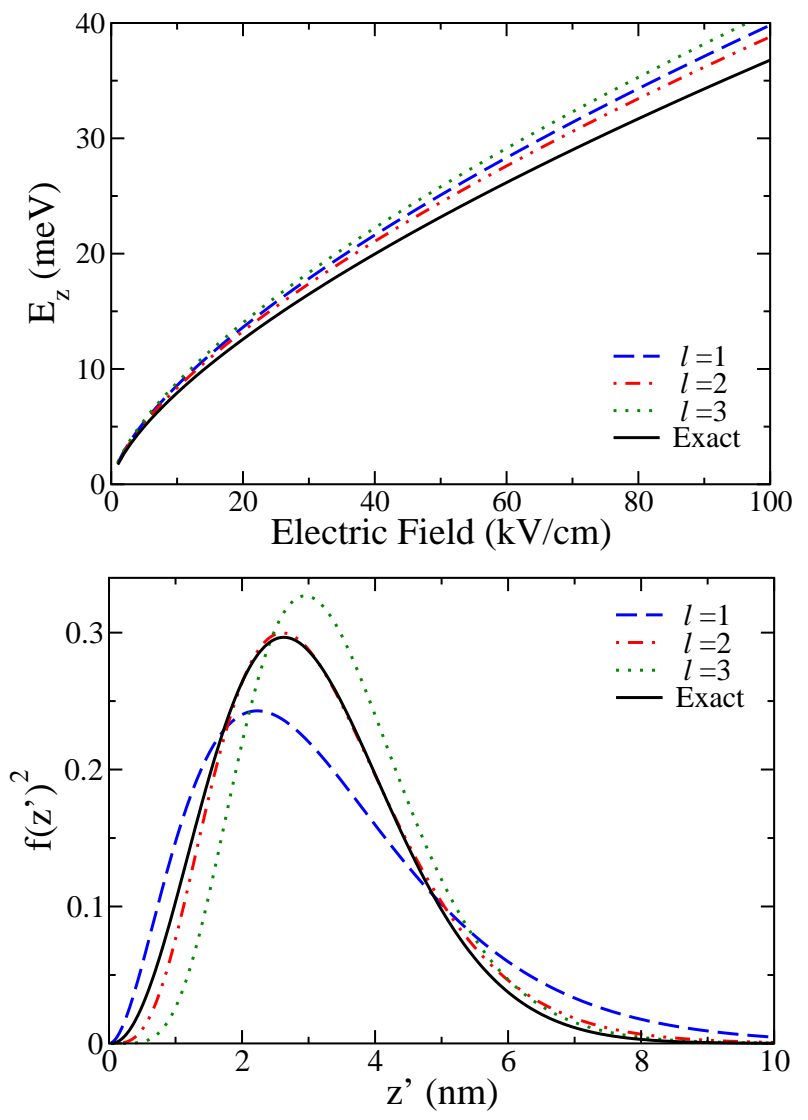

FIG. 4: (Color online) (a) $E_{z}$ for different trial functions $f(z)$ compared with the exact solution of the infinite triangular potential. ${ }^{40}$ (b) Resulting wave-function for $F=50 \mathrm{kV} / \mathrm{cm}$. $z^{\prime}=z+d$. Both the energy and wave-function for $\ell=2$ give the best match to the exact solution.

with the exact solution of an infinite triangular well $1^{40}$

$$
f^{\text {exact }}\left(z^{\prime}\right)=A i\left(\sqrt[3]{\frac{2 m_{z} e F}{\hbar^{2}}}\left[z^{\prime}-\frac{E_{0}}{e F}\right]\right),
$$

where $A i$ is the Airy function, $z^{\prime}=z+d$, and $E_{0}$ is the ground state energy

$$
E_{0}=E_{z}^{\text {exact }}=\sqrt[3]{\frac{\hbar^{2}}{2 m_{z}}(0.7587 \pi e F)^{2}} .
$$

The results are shown in Fig. 4 for $\ell=1,2$, and 3 . $\ell=2$ gives the best agreement with the exact solution for both the energy and the wave-function and is therefore adopted in what follows.

For the $\rho$-part we use the ansatz

$$
g(\rho)=\frac{\beta}{\sqrt{\pi}} e^{-\beta^{2} \rho^{2} / 2},
$$

with the variational parameter $\beta$ calculated by minimizing $E_{\rho}=\left\langle g(\rho)\left|T^{\rho}+V_{I}^{\rho}\right| g(\rho)\right\rangle$. We have checked that

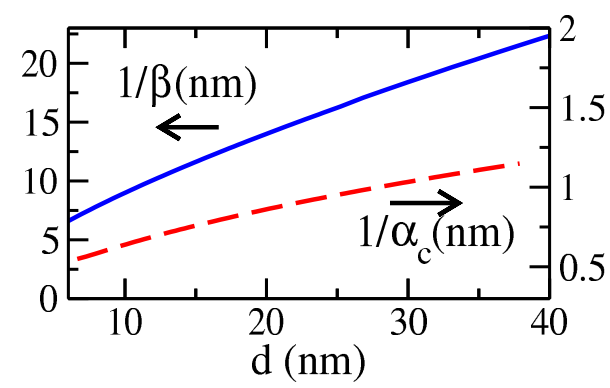

FIG. 5: (Color online) Typical confinement lengths of the interface state in the $x y$-plane $1 / \beta$ and $z$-direction $1 / \alpha . \alpha$ depends on the value of the electric field applied: $\alpha_{\mathrm{c}}$ shown here corresponds to $F_{\mathrm{c}}(d)$.

this gaussian form gives lower energy than an exponential $e^{-\eta \rho / 2}$ (as a reminiscent of the donor wave-function) for distances $d>1 \mathrm{~nm}$.

In Fig. 3(b) we plot the energy $E_{\rho}$ for the ground state and the first excited state $\left(g^{\prime}(\rho) \propto x g_{\beta^{\prime}}(\rho)\right)$ for both the variational solution adopted here and the parabolic approximation of $V_{I}^{\rho}$. The parabolic approximation gives an underestimation of the binding energies and diverges at short distances $d$ (not shown). To guarantee that the electron remains bound, and at the ground state, the operating temperature has to be lower than the energy difference between the ground and the first excited states $k_{B} T \ll \min \left(\left|E_{\rho}\right|, E_{\rho}^{\prime}-E_{\rho}\right)$. For $d=30 \mathrm{~nm}$, the excitation gap $E_{\rho}^{\prime}-E_{\rho} \sim 1 \mathrm{meV}$. This limits the operating temperature to a few Kelvin (in current experiments, temperatures as low as $0.1 \mathrm{~K}$ are being used) $\stackrel{41,42}{\underline{4}}$

The inverse of the variational parameters, $1 / \alpha$ and $1 / \beta$, are proportional to the confinement lengths in the $z$-direction and the $x y$-plane respectively. Both depend on the distance $d$ of the donor from the interface as shown in Fig. 5. and $1 / \alpha$ also depends on the value of the applied electric field, being larger for smaller $F$. Fig. 5 5ives the value of $1 / \alpha$ for the electric field $F_{\mathrm{c}}(d)$ at which the expectation values of the energies of the states at the interface and at the donor are degenerate. The expectation value of the position of the electron in the $z$-direction is $\int_{-d}^{\infty}(z+d) f^{2}(z) d z=5 / \alpha$ from the interface. This value is small compared to $d$ (for $d=30 \mathrm{~nm}, 5 / \alpha=5 \mathrm{~nm}$ ), justifying the validity of the two-well approach we are using to solve the Hamiltonian.

At the interface, the $z$-valleys' energy is lower than the $x y$-valleys'. This is straightforward to show for an infinite triangular potentia $\underline{40}^{40}$ in which $E_{z}\left(m_{\perp}\right) / E_{z}\left(m_{\|}\right)=$ $\gamma^{1 / 3}=1.68637$ [see Eq. (14)] . The difference between the levels depends on the electric field as $F^{2 / 3}$. $\Delta E=E_{z}\left(m_{\perp}\right)-E_{z}\left(m_{\|}\right)$is shown in Fig. 7 (a) (right axis). For a field $F=5 \mathrm{kV} / \mathrm{cm}$, which is small for our interests, the splitting is $\Delta E=2.5 \mathrm{meV}$ which corresponds to $T \sim 30 \mathrm{~K}$. If a magnetic field is applied, the 
$z$-levels increase their energy faster than the $x y$-levels until they cross. However, this crossing happens at a very large magnetic field $B>20 \mathrm{~T}$ (shown in the left axis of Fig. [7(a) as a function of the electric field $F$ ). Therefore, for the range of parameters of interest here, the $z$-valleys are always the ground state at the interface. We point out that it is experimentally established that, in MOSFET geometries equivalent to the one studied here, the interface ground state is non-degenerate, with a $0.1 \mathrm{meV}$ gap from the first excited state. $\stackrel{39}{ }$ This is well above the operation temperatures in the quantum control experiments investigated in the present context.

The magnetic field has two main effects on the states: (i) the electron gets more confined in the direction parallel to the interface and, consequently, (ii) its kinetic energy increases. The effect of the magnetic field is strongest for the less confined wave-functions, which correspond to the larger $d$ 's. We can quantify the strength of this effect by calculating the magnetic field $B_{\mathrm{c}}$ that is needed to get a magnetic length $\lambda_{B}$ of the same order of the confinement length in the plane parallel to the interface: for a donor a distance $d=30 \mathrm{~nm}, 1 / \beta=18.5 \mathrm{~nm}$ and $B_{\mathrm{c}} \sim 2 \mathrm{~T}$ while for $d=15.8 \mathrm{~nm}, 1 / \beta=12 \mathrm{~nm}$ and $B_{\mathrm{c}} \sim 4.5 \mathrm{~T}$. The confinement effect is illustrated in Fig. 6 where $1 / \beta_{B}$ as a function of magnetic field for two different values of $d$ is shown. The thick lines correspond to the variational solution of minimizing

$$
H_{\rho}=-\frac{\partial^{2}}{\partial x^{2}}-\frac{\partial^{2}}{\partial y^{2}}+\frac{1}{4} \mu^{2} \rho^{2}+\frac{2 Q}{\sqrt{\rho^{2}+d^{2}}}
$$

with trial function $\sim \exp \left(-\beta_{B}^{2} \rho^{2} / 2\right)$. Closer donors produce a larger confinement of the interface electron wavefunction but the effect of the magnetic field is much more dramatic for the donors further away from the interface: for $d=30 \mathrm{~nm}$, a magnetic field of $10 \mathrm{~T}$ decreases the wave-function radius by a $40 \%$. Within the parabolic approximation for the interface potential $V_{\text {parab }}(\rho)$ (which overestimates the wave-function confinement) the dependence of $\beta_{B}$ on the field is given by $\underline{43}$

$$
\beta_{B}=\left(\beta^{4}+1 / 4 \lambda_{B}^{4}\right)^{1 / 4},
$$

with values as shown in Fig. 6 (thin lines).

The increase in energy with magnetic field, as calculated variationally, is shown in Fig. 7 (b). We observe again that the effect of the magnetic field is much stronger for the larger values of $d$. The much smaller shift in the donor ground state energy (discussed in the next subsection) is also shown for comparison.

\section{Donor state $\psi_{D}$}

The potential $V_{D}$ consists of the isolated impurity Coulomb potential

$$
V_{D}=-\frac{2}{r}
$$

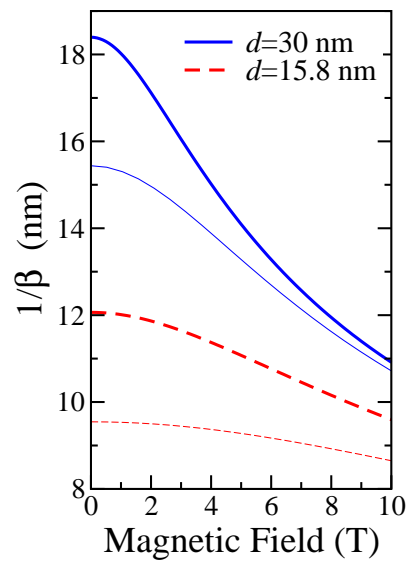

FIG. 6: (Color online) $1 / \beta$ versus magnetic field for two different interface-donor distances. Thick lines represent the variational results and the thin lines correspond to the harmonic approximation in Eq. (17).

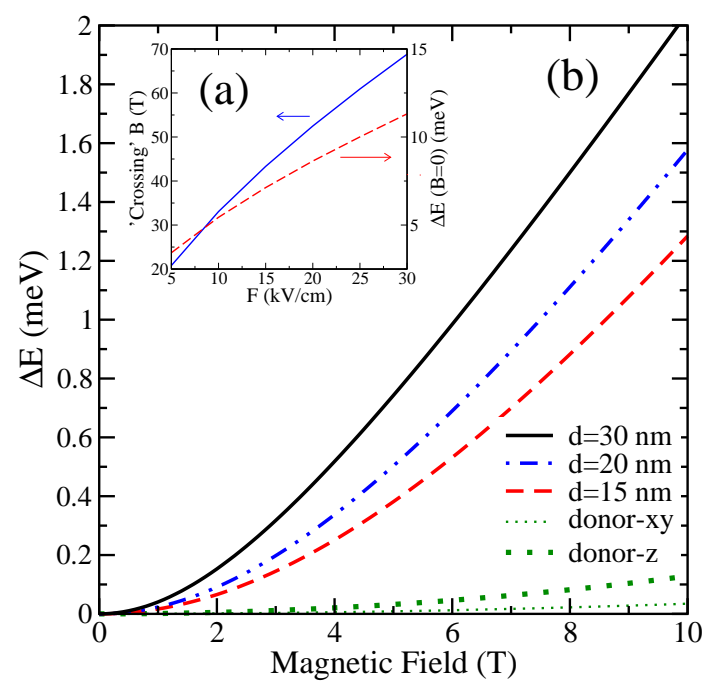

FIG. 7: (Color online) (a) (Right axis) Splitting between the $z$ - and the $x y$-levels at the interface due to the anisotropic mass. (Left axis) Magnetic field at which the $x y$-levels become the ground state at the interface. (b) Energy shift of the interface ( $z$-valleys) and donor levels (both $z$ - and $x y$-valleys) under an applied magnetic field. Due to the mass anisotropy, the shift with magnetic field for the $z$-valleys levels is larger than for the $x y$-levels. At the donor, this implies that the degeneracy of the valleys is broken. At the interface, the $z$ valleys are the ground state up to very large values of the magnetic field as shown in (a).

The solution of $H_{D}=T+V_{D}$ is taken to be of the form of the anisotropic envelope wave-function, $\underline{35}$ multiplied by $(z+d)$ to satisfy the boundary condition at the interface $\left.\psi_{D}\right|_{z=-d}=0$

$$
\psi_{D}=N(z+d) e^{-\sqrt{\rho^{2} / a^{2}+z^{2} / b^{2}}},
$$

where $1 / N^{2}=\pi a^{2} b\left(d^{2}+b^{2}-\frac{1}{2} b e^{-2 d / b}\left(\frac{1}{2} d+b\right)\right)$, for 


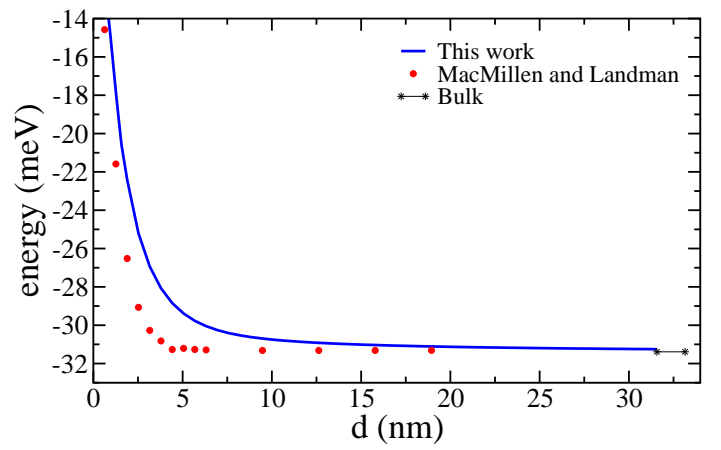

FIG. 8: (Color online) Variational donor ground state energy $\left\langle\psi_{D}\left|H_{D}\right| \psi_{D}\right\rangle$ versus $d$ obtained from our trial function $\psi_{D}$ and from the trial function used by MacMillen and Landman ${ }^{38}$ (data taken from Table III in Ref. 38). The short line at large $d$ represents the value of the ground state energy of an isolated donor in bulk $\mathrm{Si}$ (within the single valley approximation) $-31.2 \mathrm{meV}{ }^{35} \mathrm{~A}$ "perfectly imaging" plane is used $\left(\epsilon_{\text {oxide }}=\right.$ 0 ), as assumed in Ref. 38 . For the intermediate and large values of $d$ of interest here, results from the two approaches are in reasonable agreement.

$z \geq-d . \quad \psi_{D}=0$ for $z<-d$. For $d \gg a, b, \psi_{D}$ reduces to the bulk limit $\psi_{D}^{\text {bulk }}=$ $1 /\left(\pi a^{2} b\right) \exp \left(-\sqrt{\rho^{2} / a^{2}+z^{2} / b^{2}}\right) . \quad a$ and $b$ are variational parameters chosen to minimize the ground state energy. Except for the smallest distances $d<2 a^{*} \sim$ $6 \mathrm{~nm}$, not relevant here, $a$ and $b$ coincide with the KohnLuttinger variational Bohr radii of the isolated impurity $(d \rightarrow \infty) a=2.365 \mathrm{~nm}$ and $b=1.36 \mathrm{~nm}$.

In Fig. 8 we show the variational results for the ground state energy obtained from our trial function $\psi_{D}$. For comparison, we also give results obtained through the trial function proposed by MacMillen and Landman,, 38 where, aiming at a good description for donors at very short distances from the interface (typically smaller than the effective Bohr radius $a^{*}$ ), a much larger set of variational states was used for the expansion of the donor state. For this comparison, our results in Fig. 8 correspond to a "perfectly imaging" plane $\left(\epsilon_{\text {oxide }}=0\right)$, as assumed in Ref. 38. The energy depends strongly on $d$ for the smaller values of $d$, and tends to the bulk value at long distances. For the intermediate and large values of $d$ of interest here, the two approaches are essentially equivalent.

We find that the effect of the external fields on the donor state is negligible. For instance, for the largest electric fields of interest here $\left(F_{\mathrm{c}}\right.$ at short distances $d \sim 6 \mathrm{~nm}$ ), the energy corresponding to the electric field potential is $\left\langle\psi_{D}|\kappa e F z| \psi_{D}\right\rangle=0.18 R y^{*}$, to compare with $1.6 R y^{*}$ for the isolated donor ground state in bulk. The effect of a magnetic field on the electron wave-function at the donor is also very small: the field required to get a magnetic length of the order of the Bohr radius $a=2.365 \mathrm{~nm}$ is $B_{\mathrm{c}} \sim 120 \mathrm{~T}$ ! The donor ground state energy shift due to the magnetic field can be estimated

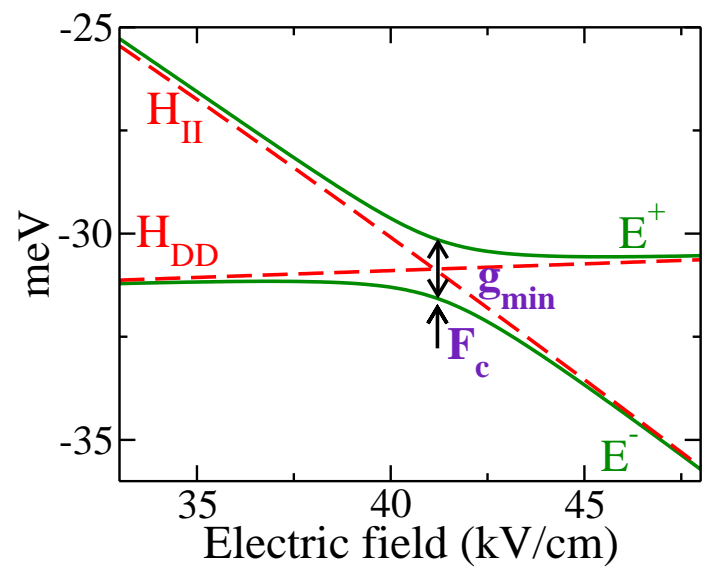

FIG. 9: (Color online) Eigenenergies $E^{+}$and $E^{-}$of the Hamiltonian in Eq. (3) for $d=11 \mathrm{~nm}$. They show an anticrossing behavior with a minimum gap $g_{\min }$ at $F_{\mathrm{c}}$. Tunneling times are related to $g_{\min }$ as $\tau=\hbar / g_{\min } . F_{\mathrm{c}}$ can be determined as the field at which $H_{I I}=H_{D D}$.

by $\underline{\underline{44}}$

$$
\Delta E=\frac{\left\langle\tilde{r}^{2}\right\rangle}{4 \lambda_{B}^{4}} R y^{*}
$$

where lengths are given in units of $a^{*},\left\langle\tilde{r}^{2}\right\rangle=2 a^{2} / a^{* 2}$ for the $z$-envelopes, and $\left\langle\tilde{r}^{2}\right\rangle=\left(a^{2}+b^{2}\right) / a^{* 2}$ for the $x y$ envelopes. The results are shown in Fig. 7(b) and are comparable to the values for the $1 \mathrm{~s}$ orbital of shallow donors calculated numerically in Ref. 45. Note that the magnetic field partially breaks the six-valley degeneracy due to the different confinement radius of the electron wave-function in each of the different valleys.

\section{Shuttling between interface and donor states}

We model the donor electron ionization under an applied electric field along $z$ by considering the tunneling process from the donor well into the triangular well at the $\mathrm{Si} / \mathrm{SiO}_{2}$ interface (see Fig. 2). The required value of the field for ionization to take place may be estimated from $H_{I I}=H_{D D}$ [see Eq. (6)]. We call $F_{\mathrm{c}}$ the characteristic field for which this condition is fulfilled, which is equivalent to require that the gap between the two eigenenergies $E^{+}$and $E^{-}$of $H$ is minimum, as illustrated in Fig. 9 .

Our results for $F_{\mathrm{c}}$ versus $d$ are shown by the solid dots [labelled (1)] in Fig. 10, In this figure we also test the robustness of our approach, namely using $\psi_{D}$ as given in Eq. (19), by comparing the values of $F_{\mathrm{c}}$ obtained assuming different forms for the donor trial function. Curves (2) and (3) correspond to isotropic and anisotropic wavefunctions respectively, with the same ground state energy for the electron at the donor as obtained from $\psi_{D}$ in Eq. (19), $\sim-31.2 \mathrm{meV}$. Note that they compare very well with curve (1). Curve (4) corresponds to a tight-binding 


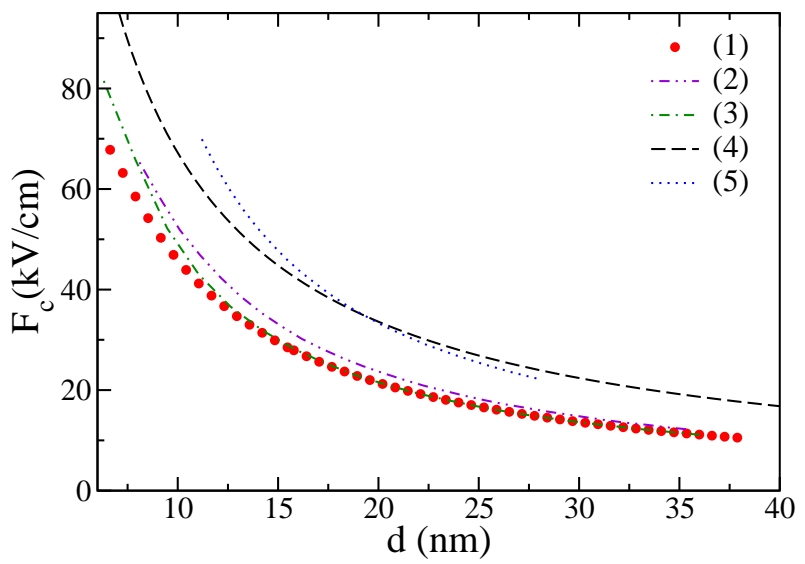

FIG. 10: (Color online) Comparison of $F_{\mathrm{c}}$ versus $d$ for the single donor problem $(\mathrm{P})$ obtained from different forms for $\psi_{D}$. Label (1) corresponds to $\psi_{D}$ with anisotropic mass as defined in Eq.(19). Label (2) assumes isotropic effective mass $m^{*}=0.29819 m$, chosen so that the ground state energy is $-31.2 \mathrm{meV}$, the same as for the single valley approximation. Label (3) corresponds to $\psi_{D} \sim \exp \left(-\sqrt{\rho^{2} / a^{2}+z^{2} / b^{2}}\right)$, and the same ground state energy. Label (4) reproduces the tight-binding results in Ref. 46, where the six valley degeneracy is considered, leading to the ground state energy of $-45 \mathrm{meV}$. The latter coincides with the experimental binding energy for a $\mathrm{P}$ donor in Si. Label (5) considers $\psi_{D} \sim \exp (-r / a)$ with isotropic mass $m^{*}=0.43 m$ so that the ground state energy is $-45 \mathrm{meV}$.

result $\frac{46}{6}$ in which the six-valley degeneracy of Si is incorporated. Although all curves are qualitatively similar, curve (4) is shifted towards larger fields. The origin of this shift is investigated by considering an isotropic trial function whose parameters have been chosen to give a ground state energy $\sim-45 \mathrm{meV}$, and we note that the results, given in curve (5), compare very well with those in curve (4). We conclude that the shift in the value of $F_{\mathrm{c}}$ when the six-fold degeneracy of the Si conduction band is considered is mainly due to the fact that the ground state energy at the donor in the single valley approximation [curve (1)] is $\sim-31.2 \mathrm{meV}$ while it is $\sim-45 \mathrm{meV}$ when the intervalley coupling is included [curve (4)]. In the following we use $\psi_{D}$ as defined in Eq. (19), keeping in mind that electric field values are bound to be somewhat underestimated.

We may picture the electron shuttling between the two wells under applied electric and magnetic fields by calculating the expectation value of its position along $z$ at the ground state, $\langle z\rangle=\left\langle\Psi^{-}|z| \Psi^{-}\right\rangle$. The results for $d=20.5 \mathrm{~nm}$ are shown in Fig. 11, where the horizontal lines mark the position of the interface. The distance between $\langle z\rangle$ and the interface tends to $5 / \alpha$ for $F \geq F_{\mathrm{c}}$, where $\alpha$ also depends on $F$. In Fig. [11(a) we show pictorially how the electron would evolve from the donor to the interface well when an electric field is applied.

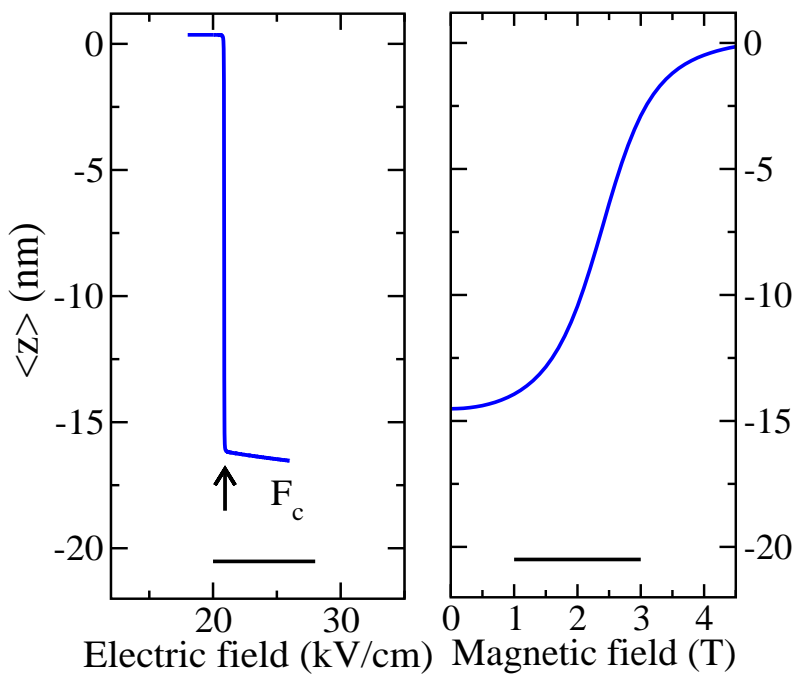

FIG. 11: (Color online) Expectation value of the electron $z$-coordinate $\langle z\rangle=\left\langle\Psi^{-}|z| \Psi^{-}\right\rangle$for $d=20.5 \mathrm{~nm}$ versus (a) electric field and (b) magnetic field $\left[F=F_{\mathrm{c}}+60 \mathrm{~V} / \mathrm{cm}\right]$. The horizontal lines represent the relative position of the interface. The donor is at $\langle z\rangle=0$. The electric field moves the electron from the donor to the interface while a parallel magnetic field takes the electron back to the donor.

At small values of $F$ the electron is at the donor well, $\langle z\rangle \sim 0$ and $\Psi^{-} \approx \psi_{D}$. The center of mass is slightly shifted from the donor site due to the factor $(z+d)$ in $\psi_{D}$. Above $F_{\mathrm{c}}$, the electron eventually tunnels to the interface. Starting with the electron at the interface in a near-degeneracy configuration $\left(F \gtrsim F_{\mathrm{c}}\right)$, a relatively modest magnetic field can cause the electron to move in a direction parallel to the field and against the electric field, as shown in Fig. 11(b). This is due to the much larger shift of the interface state energy with magnetic field compared to the shift of the donor ground state energy [see Fig. (7(b)]. This behavior characterizes electrons originating from the donors, and not other charges that the SET may detect, like charges in metallic grains on the device surface $\stackrel{42}{\underline{4}}$ The combination of parallel electric and magnetic fields constitutes therefore a valuable experimental setup to investigate whether charge detected at the interface actually originates from a donor $\underline{34}$

A key parameter determining the feasibility of quantum computation in the doped $\mathrm{Si}$ architecture is the time required to shuttle the electron between the donor and the interface. This time should be orders of magnitude smaller than the coherence time to allow for many operations and error correction while coherent evolution of the qubit takes place. The tunneling process conserves the spin, but coherence would be lost for orbital/charge degrees of freedom. Therefore, if quantum information is stored in a charge qubit, the electron should evolve adiabatically from the donor to the interface, while tunneling would be acceptable for spin qubits. In an adiabatic process the modification of the Hamiltonian (for instance, 


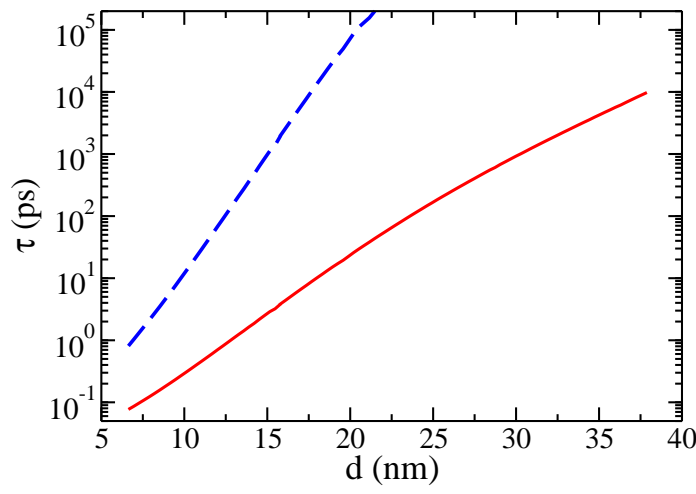

FIG. 12: (Color online) Donor ionization tunneling (solid line) and adiabatic passage (dashed line) times versus $d$.

when an external field is applied) is slow enough that the system is always in a known energy eigenstate, going continuously from the initial to the final eigenstate. $\stackrel{47}{*}$ Here, we calculate both tunneling and the adiabatic passage times.

We estimate the tunneling time from the minimum gap $g_{\text {min }}$ between the two eigenvalues $E^{+}$and $E^{-}$(see Fig. 9) via the uncertainty relation $\tau=\hbar / g_{\min }$. The adiabatic time is calculated as $\frac{46.48}{4} \tau_{a}=\hbar|e| F_{\max } d / g_{\min }^{2}$ and is orders of magnitude larger than the tunneling time. $F_{\max }$ is chosen so that the electron is at the interface $\Psi^{-} \approx \psi_{I}$. The results for the tunneling and adiabatic passage times (for $F_{\max }=2 F_{\mathrm{c}}$ ) are shown in Fig. 12. The times depend exponentially on the distance $d$. Tunneling times range from $0.1 \mathrm{ps}$ for $d=6 \mathrm{~nm}$ to $10 \mathrm{~ns}$ for $d=38 \mathrm{~nm}$. Adiabatic times range from $1 \mathrm{ps}$ for $d=6 \mathrm{~nm}$ to $100 \mathrm{~ns}$ for $d=20 \mathrm{~nm}$ and get very large at longer distances. These times are to be compared to the experimental values of spin coherence and charge coherence respectively.

Spin dephasing in $\mathrm{Si}$ is mainly due to dipolar fluctuations in the nuclear spins in the system, which produce a random magnetic field at the donor electron spin. The spin dephasing time in bulk natural $\mathrm{Si}$ is $T_{2} \sim 1 \mathrm{~ms}, \underline{2}, \underline{4}, 5, \underline{6}$ Natural Si is mostly composed of ${ }^{28} \mathrm{Si}$ (no nuclear spin), with a small fraction $\left(4.67 \%\right.$ ) of ${ }^{29} \mathrm{Si}$ (nuclear spin 1/2), therefore, $T_{2}$ can be dramatically improved through isotope purification $2,4,5,6$ up to $100 \mathrm{~ms}$ or longer in bulk. Moreover, it has been recently proposed that the spin dephasing times can be arbitrarily prolonged by applying designed electron spin resonance pulse sequences ${ }^{7}$ On the other hand, the closeness of a surface or interface can reduce the spin dephasing times $\underline{49}$ The tunneling time calculated here is orders of magnitude smaller than the bulk $T_{2} \sim 1 \mathrm{~ms}$ for natural Si: In the worst case scenario (long distances $d \sim 40 \mathrm{~nm}$ ) $T_{2} / \tau \sim 100$. Therefore, we expect tunneling times to be always much shorter than decoherence times even in the presence of an interface.

Charge coherence time is considerably shorter than spin coherence time, since charge couples very strongly to the environment through the long range Coulomb interaction. The main channels of decoherence are charge

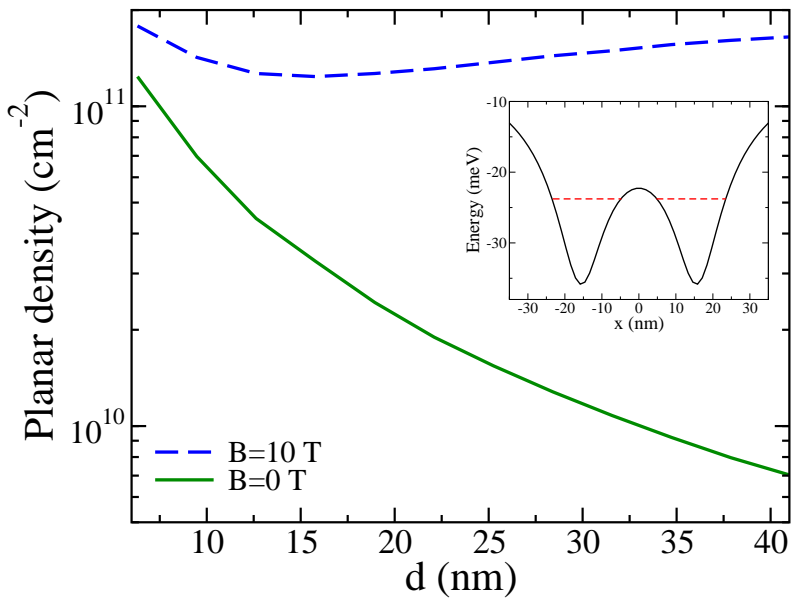

FIG. 13: (Color online) The main panel gives the maximum donor planar density $n_{\max }$ for which electrons drawn to the interface remain localized around the donor region, and do not form a 2DEG. It is assumed that all donors are at the same distance from the interface $d . n_{\max }$ is estimated from the criterion that the barrier between neighboring wells at the interface is above $E_{\mathrm{DW}}$ given in Eq. (22). Results are shown for $B=0 \mathrm{~T}$ and $B=10 \mathrm{~T}$. The inset shows the double well potential parallel to the interface created by two donors located a distance $d=6.3 \mathrm{~nm}$ from the interface and separated by $R=28 \mathrm{~nm}$. The expectation value of the energy $E_{\mathrm{DW}}$ (given by the dashed lines for $B=0$ ) is also lower than the single-donor well ground state energy.

fluctuations and electron-phonon interactions $, 50,51$ The charge coherence time has been measured to be $\sim 200 \mathrm{~ns}$ for Si quantum dots surrounded by oxide layers $\underline{20}$ This number has to be compared to the calculated adiabatic times, which are much longer than the tunneling times. Therefore, for charge qubits to be realizable in the configuration discussed here, the donor-interface distance $d$ has to be limited to a maximum of $20 \mathrm{~nm}$.

\section{DONOR PAIR}

\section{A. Planar density}

We estimate the maximum planar density of donors $\left(n_{\max }\right)$ allowed to avoid the formation of a $2 \mathrm{DEG}$ as $n_{\max }=1 / R_{\min }^{2}$, where $R_{\min }$ is a minimum distance between two donors which is calculated as follows: We assume two donors located at the same distance $d$ from the interface and a distance $R$ apart. The resulting double well potential along the interface $x y$-plane is

$$
\begin{aligned}
V_{\mathrm{DW}}(x, y) & =\frac{2(Q-1)}{\sqrt{(x+R / 2)^{2}+y^{2}+d^{2}}} \\
& +\frac{2(Q-1)}{\sqrt{(x-R / 2)^{2}+y^{2}+d^{2}}},
\end{aligned}
$$


as illustrated in the inset of Fig. 13 for $d=6.3 \mathrm{~nm}$ and $R=28 \mathrm{~nm}$. We adopt two different criteria to estimate $R_{\min }$. (i) The first one requires $R_{\min }=2 / \beta$ where $2 / \beta$ is the width of the gaussian $g(\rho)$ (see Fig. 5). For instance, for $d=30 \mathrm{~nm}$, this gives $R_{\min } \approx 40 \mathrm{~nm}$ leading to $n_{\max } \approx$ $6 \times 10^{10} \mathrm{~cm}^{-2}$, while for $d=10 \mathrm{~nm}, R_{\min } \approx 18 \mathrm{~nm}$ and $n_{\max } \approx 3 \times 10^{11} \mathrm{~cm}^{-2}$. (ii) The second criterion, which we find to be slightly more restrictive, requires a high enough barrier within the double well, and is given by

$$
E_{\mathrm{DW}}=\left\langle\psi_{I}\left|H_{\mathrm{DW}}\right| \psi_{I}\right\rangle \leq V_{\mathrm{DW}}(0,0)
$$

where $R_{\text {min }}$ corresponds to the equality condition. $H_{\mathrm{DW}}$ is the double-well 2-dimensional Hamiltonian

$$
H_{\mathrm{DW}}=T_{x}+T_{y}+V_{\mathrm{DW}}(x, y)
$$

with $T_{x}$ and $T_{y}$ the kinetic energy terms. $V_{\mathrm{DW}}(0,0)$ is the maximum height of the inter-well barrier, which is required to be above the single-particle expectation value of the energy $E_{\mathrm{DW}}$. The maximum planar density estimated from Eq. (22) is shown in the main panel of Fig. 13. For instance, $n_{\max }(d=30 \mathrm{~nm}) \approx 10^{10} \mathrm{~cm}^{-2}$ obtained from $R_{\min } \approx 88 \mathrm{~nm}$. $n_{\max }$ is larger for the donors closer to the interface. For instance, $n_{\max }(d=$ $10 \mathrm{~nm}) \approx 6 \times 10^{10} \mathrm{~cm}^{-2}\left(R_{\min } \approx 38 \mathrm{~nm}\right)$.

As shown in Fig. 6, $1 / \beta_{B}$ decreases with a perpendicular magnetic field, hence increasing the maximum planar density. The first criterion for the maximum planar density gives, for $d=30 \mathrm{~nm}$ and $B=10 \mathrm{~T}, R_{\min } \approx 22 \mathrm{~nm}$ and $n_{\max } \approx 2 \times 10^{11} \mathrm{~cm}^{-2}$. The second criterion gives the dashed curve in Fig. 13 which is, on average, almost one order of magnitude larger than without a magnetic field. Note that the effect of the magnetic field is much stronger for large distances $d$.

\section{B. Qubit interaction at the interface: exchange}

One of the problems for quantum computation in doped Si arises from the lack of control of the exact position of the donors. The main consequence of this is the indetermination of the value of the exchange between two neighboring donor electrons due to the theoretically predicted oscillations of exchange with $R$, caused by valley interference effects ${ }^{8}, 32$ One straightforward way to alleviate this problem is to perform these operations at the interface ${ }^{34}$ where, as discussed in Sec. IIB, this degeneracy is lowered. Additionally, it would be much easier to control the qubit operations when the electrons are at the interface, similar to the successful experiments on double quantum dots in $\mathrm{GaAs}^{28,29,30,31}$ and $\mathrm{Si}^{20}$ Note that the potential created by donor pairs (inset in Fig. 13) resembles very much a double quantum dot with the clear advantage that, in this case, the potential is produced exclusively by the Coulomb attraction of the donors and its exact form is known: $V=2(Q-1) / \sqrt{\rho^{2}+d^{2}}$.

It is therefore of interest to determine the exchange coupling between donor electrons at the interface. As

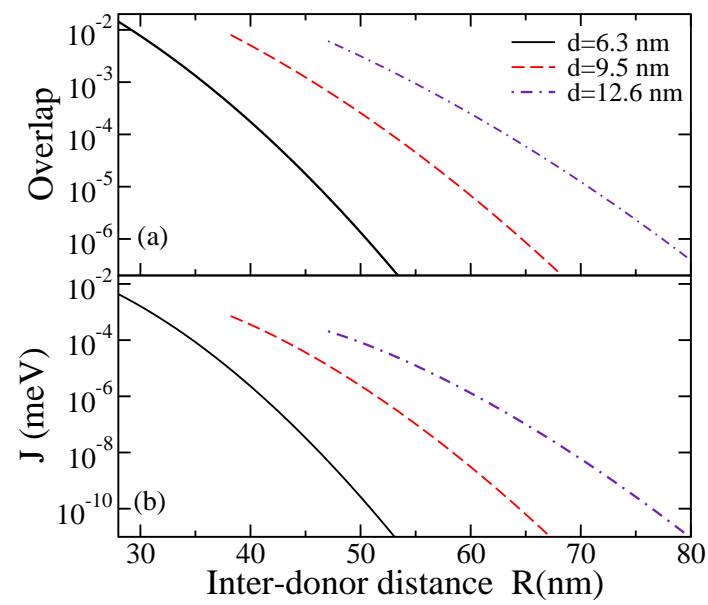

FIG. 14: (Color online) (a) Overlap $S$ between electron wavefunctions at neighboring wells at the interface for three different values of $d$. (b) Exchange $J$ calculated within the HeitlerLondon approximation. Values for $S$ and $J$ given here satisfy $n<n_{\max }$.

a first approach, we perform these calculations within the Heitler-London method. The validity and limitations of this method to calculate exchange in semiconductor nanostructures has been previously discussed by the authors. ${ }^{52}$ The expression for the exchange within this approximation is

$$
\begin{aligned}
J & =\frac{2 S^{2}}{1-S^{4}}\left\langle\Phi_{L}(1) \Phi_{R}(2)\left|2 H_{\mathrm{DW}}(1)+\frac{e^{2}}{\epsilon_{\mathrm{Si}} r_{12}}\right| \Phi_{L}(1) \Phi_{R}(2)\right\rangle \\
& -\frac{2}{1-S^{4}}\left\langle\Phi_{L}(1) \Phi_{R}(2)\left|2 H_{\mathrm{DW}}(1)+\frac{e^{2}}{\epsilon_{\mathrm{Si}} r_{12}}\right| \Phi_{L}(2) \Phi_{R}(1)\right\rangle
\end{aligned}
$$

where $\Phi_{L, R}=g(x \mp R / 2, y), S=\exp \left[-\beta^{2}(R / 2)^{2}\right]$ is the overlap, and $e^{2} / \epsilon_{\mathrm{Si}} r_{12}$ is the electron-electron interaction with $r_{12}$ the distance between electron (1) and electron (2). The first term in Eq. (24) is the direct term and the second is the exchange term.

In Fig. 14 we show the exchange $J$ and the overlap $S$ versus the inter-donor distance $R$ for three different values of $d$. Note that the overall dependence of these two quantities with $R$ is very similar, indicating that the behavior of $J$ is closely related to the overlap. $52 J$ and $S$ values are shown only for distances $R>R_{\text {min }}$, defined in Sec. IIIA For a wide range of $R$ 's, $J$ is of the same order as in GaAs double quantum dots where $J$ 's as low as $\sim 10$ neV have been measured. 31

Exchange control can be performed by applying a magnetic field perpendicular to the interface, which reduces the wave-function radius, therefore decreasing the overlap. The effect of the magnetic field on the exchange is well known: ${ }^{15} J$ decreases and eventually changes sign when the triplet is favored becoming the ground state. This is illustrated in Fig. 15 for $d=6.3 \mathrm{~nm}$ and $R=28 \mathrm{~nm}$. At very large fields the singlet and triplet 


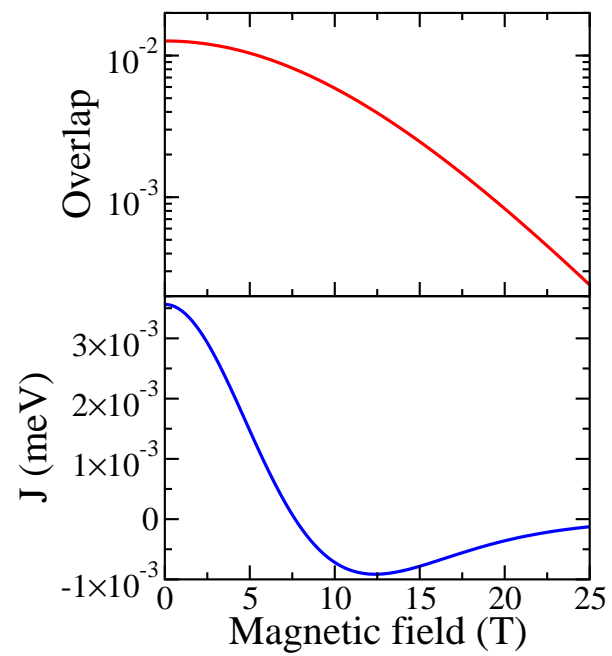

FIG. 15: (Color online) Modulation of overlap and exchange by a magnetic field perpendicular to the interface in the particular case $d=6.3 \mathrm{~nm}$ and $R=28 \mathrm{~nm}$. The double well potential for these particular values of $d$ and $R$ is depicted in the inset of Fig. 13 .

states become degenerate, as expected. Note that, in this case, the qualitative behavior of $S$ and $J$ with magnetic field is very different.

\section{SUMMARY AND CONCLUSIONS}

Quantum computer architectures based on semiconductor nanostructure qubits have the key potential advantage of scalability, which has led to the great deal of current interest in Si- and GaAs-based quantum computation. Silicon based spin qubits have the important additional advantage of extremely long spin coherence times $\left(T_{2}^{\mathrm{Si}} \sim\right.$ miliseconds or more) since isotopic purification (eliminating ${ }^{29} \mathrm{Si}$ nuclei) could considerable suppress spectral diffusion induced electron spin decoherence $2,3,4,5,6,7$ leading to $T_{2}^{\mathrm{Si}} \sim 100 \mathrm{~ms}$, whereas electron spin coherence time is constrained to be rather short in GaAs quantum dot structures, $T_{2}^{\mathrm{GaAs}} \sim 1-10 \mu \mathrm{s}$, since neither $\mathrm{Ga}$ nor As nuclei have zero nuclear angular momentum isotopes. However, the experimental progress in Si spin qubits has been very slow whereas there has been impressive recent experimental progress in the GaAs quantum dot spin qubits $23,28,29,30,31$ The main experimental advantage of GaAs quantum dot system has been the ease in the 1-qubit and 2-qubit manipulation because the electrons near the surface can be effectively controlled by surface gates. By contrast, Si:P qubits are in the bulk, severely hindering experimental progress since electron manipulation in the bulk has turned out to be a difficult task.
In this paper we show through detailed quantitative theoretical work how to control and manipulate qubits (i.e. both single electrons and two-electron exchange coupling) in a doped-Si quantum computing architecture ${ }^{1}$ by applying external electric and magnetic fields. In particular, we have analyzed three main issues: (i) the times involved in the donor electron 'shuttling' between the donor and the interface of $\mathrm{Si}$ with (typically) $\mathrm{SiO}_{2}$ have been found to be a few orders of magnitude shorter than the spin coherence times in $\mathrm{Si}$, as required to allow for the necessary 'logic operations' and 'error correction' to take place; (ii) the existence of a well defined interface state where the electron remains bound and localized, so that it does not spread and form a 2DEG. This condition, which guarantees that electrons actually involved in a particular operation be taken back from the interface to donor sites, leads to a lower bound for the interdonor spacing, and consequently a maximum donor planar density; and (iii) the possibility of performing the two-qubit exchange gate operations at the interface, instead of around the donor sites as originally proposed $\underline{\underline{1}}$ Our results show that sufficiently large values of exchange coupling $\left(\sim 10^{-4} \mathrm{meV}\right)$ can be achieved.

Interface operations have several potential advantages over bulk operations, the most obvious one being that the read-out procedure would be simplified. A well known limitation of exchange gates for donor electrons in the bulk is the oscillatory behavior of the exchange coupling, which is due to the strong pinning of the six conduction band Bloch-function phases at each donor site, where the Coulomb potential is infinitely attractive. 53 This condition is alleviated at the interface in two ways: first, the six valley degeneracy is partially lifted and, second, although the electrons at the interface remain bound to the donors, the binding potential is not singular; it is actually equivalent to a quantum dot potential. Experiments on charge-qubit control in a double quantum dot at $\mathrm{Si}$ surface ${ }^{20}$ indicate that the exchange oscillatory behavior may not be a severe problem for donor-bound electrons manipulated at the $\mathrm{Si} / \mathrm{SiO}_{2}$ interface.

Our proposal combines the advantages of Si spin qubits (i.e. long $T_{2}$ time) with the structural advantages of GaAs qubit control and manipulation. We believe that the specific experiments we propose (and analyze in quantitative details) in this paper, if carried out, will go a long way in establishing the feasibility of a Si quantum computer.

\section{Acknowledgments}

This work is supported by LPS and NSA. B.K. also acknowledges support from CNPq, FUJB, Millenium Institute on Nanotechnology - MCT, and FAPERJ. 
1 B. E. Kane, Nature 393, 133 (1998).

2 R. de Sousa and S. Das Sarma, Phys. Rev. B 68, 115322 (2003).

3 A. M. Tyryshkin, S. A. Lyon, A. V. Astashkin, and A. M. Raitsimring, Phys. Rev. B 68, 193207 (2003).

4 E. Abe, K. Itoh, J. Isoya, and S.Yamasaki, Phys. Rev. B 70, 033204 (2004).

5 W. M. Witzel, R. de Sousa, and S. Das Sarma, Phys. Rev. B 72, 161306 (2005).

6 A. M. Tyryshkin, J. J. L. Morton, S. C. Benjamin, A. Ardavan, G. A. D. Briggs, J. W. Ager, and S. A. Lyon, Journal of Physics: Condensed Matter 18, S783 (2006).

7 W. M. Witzel and S. Das Sarma, cond-mat/0604577 (2006).

8 B. Koiller, X. Hu, and S. Das Sarma, Phys. Rev. Lett, 88, 027903 (2002).

9 B. E. Kane, MRS Bulletin 30, 105 (2005).

10 T. Schenkel, A. Persaud, S. J. Park, J. Nilsson, J. Bokor, J. A. Liddle, R. Keller, D. H. Schneider, D. W. Cheng, and D. E. Humphries, J. Appl. Phys. 94, 7017 (2003).

11 T. Shinada, S. Okamoto, T. Kobayashi, and I. Ohdomary, Nature 437, 1128 (2005).

12 D. N. Jamieson, C. Yang, T. Hopf, S. M. Hearne, C. I. Pakes, S. Prawer, M. Mitic, E. Gauja, S. E. Andresen, F. E. Hudson, et al., Appl. Phys. Lett. 86, 202101 (2005).

13 J. L. O'Brien, S. R. Schofield, M. Y. Simmons, R. G. Clark, A. S. Dzurak, N. J. Curson, B. E. Kane, N. S. McAlpine, M. E. Hawley, and G. W. Brown, Phys. Rev. B 64, 161401 (2001).

14 S. R. Schofield, N. J. Curson, M. Y. Simmons, F. J. Rueß, T. Hallam, L. Oberbeck, and R. G. Clark, Phys. Rev. Lett. 91, 136104 (2003).

15 D. Loss and D. P. DiVincenzo, Phys. Rev. A 57, 120 (1998).

16 R. Vrijen, E. Yablonovitch, K. Wang, H.-W. Jiang, A. Balandin, V. Roychowdhury, T. Mor, and D. DiVincenzo, Phys. Rev. A 62, 012306 (2000).

17 J. Levy, Phys. Rev. A 64, 052306 (2001).

18 M. Friesen, P. Rugheimer, D. E. Savage, M. G. Lagally, D. W. van der Weide, R. Joynt, and M. A. Eriksson, Phys. Rev. B 67, 121301 (2003).

19 L. C. L. Hollenberg, A. S. Dzurak, C. Wellard, A. R. Hamilton, D. J. Reilly, G. J. Milburn, and R. G. Clark, Phys. Rev. B 69, 113301 (2004).

20 J. Gorman, D. G. Hasko, and D. A. Williams, Phys. Rev. Lett. 95, 090502 (2005).

21 D. Rugar, R. Budakian, H. J. Mamin, and B. W. Chui, Nature 430, 329 (2004).

${ }^{22}$ M. Xiao, I. Martin, E. Yablonovitch, and H. Jiang, Nature 430, 435 (2004).

${ }^{23}$ F. H. L. Koppens, C. Buizert, K. J. Tielrooij, I. T. Vink, K. C. Nowack, T. Meunier, L. P. Kouwenhoven, and L. M. K. Vandersypen, Nature 442, 766 (2006).

${ }^{24}$ L. C. L. Hollenberg, C. J. Wellard, C. I. Pakes, and A. G. Fowler, Phys. Rev. B 69, 233301 (2004).

25 A. D. Greentree, A. R. Hamilton, L. C. L. Hollenberg, and R. G. Clark, Phys. Rev. B 71, 113310 (2005).

26 A. R. Stegner, C. Boehme, H. Huebl, M. Stutzmann, K. Lips, and M. S. Brandt, Nat. Phys. 2, 835 (2006).

27 M. Calderón, B. Koiller, and S. Das Sarma, cond- mat/0610089 (2006).

28 J. R. Petta, A. C. Johnson, J. M. Taylor, E. A. Laird, A. Yacoby, M. D. Lukin, C. M. Marcus, M. P. Hanson, and A. C. Gossard, Science 309, 2180 (2005).

${ }^{29}$ F. H. L. Koppens, J. A. Folk, J. M. Elzerman, R. Hanson, L. H. W. van Beveren, I. T. Vink, H. P. Tranitz, W. Wegscheider, L. P. Kouwenhoven, and L. M. K. Vandersypen, Science 309, 1346 (2005).

30 A. C. Johnson, J. R. Petta, J. M. Taylor, A. Yacoby, M. D. Lukin, C. M. Marcus, M. P. Hanson, and A. C. Gossard, Nature 435, 925 (2005).

31 E. A. Laird, J. R. Petta, A. C. Johnson, C. M. Marcus, A. Yacoby, M. P. Hanson, and A. C. Gossard, Phys. Rev. Lett. 97, 056801 (2006).

32 B. Koiller, X. Hu, and S. Das Sarma, Phys. Rev. B 66, 115201 (2002).

33 M. J. Calderón, B. Koiller, X. Hu, and S. Das Sarma, Phys. Rev. Lett. 96, 096802 (2006).

34 M. J. Calderón, B. Koiller, and S. Das Sarma, Phys. Rev. B 74, 081302 (2006).

35 W. Kohn, Solid State Physics Series, vol. 5 (Academic Press, 1957), edited by F. Seitz and D. Turnbull.

${ }^{36}$ F. Stern and W. Howard, Phys. Rev. 163, 816 (1967).

37 B. G. Martin and R. F. Wallis, Phys. Rev. B 18, 5644 (1978).

38 D. B. MacMillen and U. Landman, Phys. Rev. B 29, 4524 (1984).

39 T. Ando, A. Fowler, and F. Stern, Rev. Mod. Phys. 54, 437 (1982).

40 F. Stern, Phys. Rev. B 5, 4891 (1972).

41 A. J. Ferguson, V. C. Chan, A. R. Hamilton, and R. G. Clark, Appl. Phys. Lett. 88, 162117 (2006).

${ }^{42}$ K. R. Brown, L. Sun, and B. E. Kane, Appl. Phys. Lett. 88, 213118 (2006).

43 L. Jacak, P. Hawrylak, and A. Wójs, Quantum Dots (Springer-Verlag, Berlin, 1998).

44 L. Landau and E. Lifshitz, Quantum Mechanics (Pergamon, 1977).

45 A. Thilderkvist, M. Kleverman, G. Grossmann, and H. Grimmeiss, Phys. Rev. B 49, 14270 (1994).

46 A. S. Martins, R. B. Capaz, and B. Koiller, Phys. Rev. B 69, 085320 (2004).

47 A. Messiah, Quantum Mechanics (John Wiley \& Sons, Inc, New York, 1962).

48 F. Ribeiro, R. Capaz, and B. Koiller, Appl. Phys. Lett. 81, 2247 (2002).

49 T. Schenkel, A. M. Tyryshkin, R. de Sousa, K. B. Whaley, J. Bokor, J. A. Liddle, A. Persaud, J. Shangkuan, I. Chakarov, and S. A. Lyon, Appl. Phys. Lett. 88, 112101 (2006)

50 T. Hayashi, T. Fujusawa, H. D. Cheong, Y. H. Jeong, and Y. Hirayama, Phys. Rev. Lett. 91, 226804 (2003).

51 X. Hu, B. Koiller, and S. Das Sarma, Phys. Rev. B 71, 235332 (2005).

52 M. J. Calderón, B. Koiller, and S. Das Sarma, Phys. Rev. B 74, 045310 (2006).

53 B. Koiller, R. B. Capaz, X. Hu, and S. Das Sarma, Phys. Rev. B 70, 115207 (2004). 